\title{
Dual training as clinician-scientist in child and adolescent psychiatry: are we there yet?
}

\author{
Alexis Revet ${ }^{1}$. Johannes Hebebrand ${ }^{2} \cdot$ Sampada Bhide $^{3}$. João Caseiro ${ }^{4}$ Eugenia Conti ${ }^{5} \cdot$ Marike Deutz $^{6}$. \\ Andra ssac $^{7}$. Athanasios Kanellopoulos ${ }^{8} \cdot$ Tuğba Kalyoncu $^{9} \cdot$ Katri Maasalo $^{10}$. Silvana Markovska-Simoska ${ }^{11}$. \\ Marija Mitkovic-Voncina ${ }^{12}$. Silvia Molteni ${ }^{13} \cdot$ Mariela Mosheva $^{14}$. Susanne Mudra ${ }^{15}$. Julia Philipp ${ }^{16}$. \\ Cecil Prins-Aardema ${ }^{17} \cdot$ Marina Romero Gonzalez ${ }^{18}$. Antonín Šebela ${ }^{19} \cdot$ Jochen Seitz $^{20} \cdot$ Lise Eilin Stene $^{21}$. \\ Nikolina Vrljičak Davidović ${ }^{22} \cdot$ Ida Wessing $^{23} \cdot$ Paul Klauser $^{24}$
}

Published online: 9 January 2018

c) Springer-Verlag GmbH Germany, part of Springer Nature 2017

\begin{abstract}
"Clinician-scientist" is a term used to designate specialists who engage in both clinical and research activities, including biomedical, epidemiological or clinical research [1]. In recent years, a general decline has been observed in the number of medical trainees who choose to combine patient care and a scientific career [2]. Many factors have been proposed to explain this decline: economic constraints, duration of training, complexity of techniques used in biomedical research (from neuroimaging and genetics to informatics), and rapid pace of advances [3], but also complexity of the care for patients, cost reductions, increasing workload, and social changes (personal interest, level of pay, quality of life, and work-life balance in particular). At a deeper level, some authors have even questioned the possibility to reconcile research and clinical activity [4].

Of course, research in Child and Adolescent Psychiatry (CAP) is important to deepen our understanding of mental disorders and to improve their diagnosis, treatment, and prevention. However, there is often a gap between researchers and clinicians, between animal models and patients, and between wet labs and hospitals, that impedes the correct and smooth translation between basic research and clinical practice [5]. In this context, there is still a strong need for physicians who can transform clinical questions into research hypotheses. On the other hand, we need physicians who understand cutting-edge research to integrate new findings into evidence-based clinical practice. Furthermore, for clinicians, research represents a unique way to step back from their daily clinical practice and to challenge it.
\end{abstract}

Alexis Revet

revet.a@chu-toulouse.fr

Extended author information available on the last page of the article
Given the difficulties mentioned above, the question that arises is how we could help young trainees to accept and maintain the dual role of a clinician-scientist (not to mention the teaching part and administrative duties)?

The 2017 ESCAP Research Academy Geneva Workshop, which took place from July 9 to 11, prior to the 17th ESCAP International Congress in Geneva, gathered 23 early career investigators (19 medical doctors and 4 psychologists) aged 28-42 years, who originated from 17 European countries and 2 non-European countries (Israel and Australia) [6]. It thus offered a good opportunity to interview a sample of young clinician-scientists. They filled out a semi-structured questionnaire focusing on the main issues related to clinician-scientist training: major topics and methodological approach of research, time devoted to research, specific national training program and available support, major funding sources, wishes, and desired help, and importance of European collaboration.

Among the main difficulties encountered by these young clinician-scientists, length of the advanced educational training (especially for those aiming at a full-length $\mathrm{PhD}$ training and/or even a postdoctoral experience), organization and time sharing between clinical work and research activities, and lack of funding for research were the most frequently reported. Only a third reported availability of a clinician-scientist program in their home country. All participants highlighted the importance of the European level in their career's development, but for different reasons, ranging from getting a better chance to advance their careers when living in a country where research is poorly developed to the need to create larger cross-border cohorts through European grants, and the possibility of finding a position in another European country. The difficulty of balancing family life and academic career was also highlighted, especially by female 
participants who have to manage pregnancy and maternity leave in addition to their clinical and research duties.

Interestingly, all ESCAP Research Academy fellows with a medical degree would like to pursue both research and clinical practice, which is not the case for the four fellows trained in psychology, of whom three would like to drop clinical activity on the long term. Most of the fellows aim to succeed in an academic career. Figure 1 is a tag cloud - a visual, stylized method that represents the occurrence of words or tags within a textual content-obtained from the semi-structured interviews of the 23 participants which highlights the most prominent terms used in their response and brings out some thematic axes: time ("time", "month", "hours", "years", "week", "day"), difficulty ("anxiety", "working", "hard", "risk”), money ("salary", "money”, "fund", "grant”, "buy”, "gain", "funding”), curriculum ("university", "PhD”, "studies", "study", "training", "career"), clinical duties ("hospital", "medical", "clinical", "clinicians"), and scientific activities ("research", "PhD”, "paper" "grants", "funding").

Several propositions can be discussed to reverse the declining number of clinician-scientists in CAP and to foster their future role. First, we propose the development of special tracks for clinician-scientists with shorter specialist training (e.g., 1 year of research can count as 1 year of residency in Switzerland; https://www.fmh.ch/bildung-siwf.html ), improved coordination between clinical work and research, for instance through short research projects relevant to curriculum of interest throughout their medical studies, protected research time, integrated clinical and research training (e.g., residency/PhD track from the International Max Planck Research School for Translational Psychiatry; http://www. imprs-tp.mpg.de/2975/residency-phd-track), peer mentoring [7] - the importance of good models being fundamental [8] - and help regarding funding (e.g., MD-PhD fellowship from the Swiss National Science Foundation; http://www. snf.ch/en/funding/careers/md-phd-programme), and grant applications. Second, more efforts should be undertaken to render the clinician-scientist as a more attractive career path, in particular, by improving the pay and social status of these positions. Third, daily practice of clinician-researchers must be facilitated by the creation of integrated regional, national, and European health research centers, in which translational research will constitute a priority [5, 9]. Other aspects could be discussed such as the necessity of valorizing less "classical" fields of research in CAP such as qualitative research and research in social studies, or the importance of international exchanges regarding research but also clinical training.

The next generation of clinician-scientists should play a key role not only to bridge the gap between medicine and science but also in teaching and mentoring future trainees and managing teams of clinicians and researchers to help them to connect and work together. They will also have to establish global collaborative clinical and research networks, such as the ESCAP Research Academy [10].

Special attention should be devoted to research infrastructure in clinical settings, that has already been perceived as less organized when psychiatry is compared to other medical specialties in some countries [11]. With lower access to research, clinical professionals in child and adolescent mental health may have less of a vote in exploring and defining new information in the field as compared to their colleagues in other medical branches. Consequently, other related scientifically strong disciplines may have more to contribute to the development of CAP than CAP itself.

In conclusion, even though clinical and scientific activities are inseparable to guarantee advances in any medical specialty, the dual career in CAP is faced with certain challenges. Efforts to overcome the difficulties and improve the connections between these two interdependent professional facets may serve as an important corner-stone of increasing the involvement of CAP in its own future academic development.
Fig. 1 Tag cloud obtained from the semi-structured interviews of the 23 ESCAP Research Academy Geneva Workshop young clinician-scientists. The open access software Word Cloud Generator (Copyright $\odot$ Jason Davis) was used to generate this tag cloud with the following settings: numbers of words: 1000 ; scale: $\log \mathrm{n}$; orientation: from $-60^{\circ}$ to $+60^{\circ}$; and spiral: rectangular

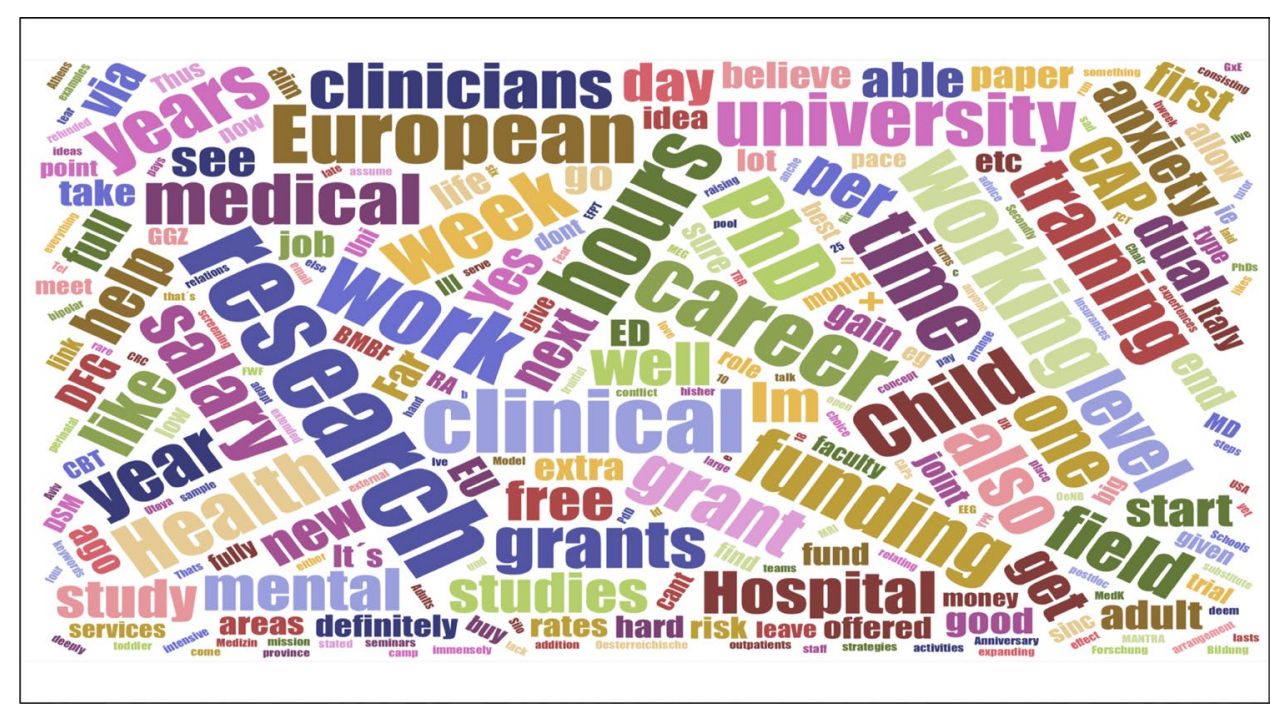




\section{References}

1. Baumal R, Benbassat J, Van JAD (2014) Reflections on the current and future roles of clinician-scientists. Isr Med Assoc J $16: 475-478$

2. Eley DS, Wilkinson D (2015) Building a teaching-research nexus in a research intensive university: rejuvenating the recruitment and training of the clinician scientist. Med Teach 37:174-180. https:// doi.org/10.3109/0142159X.2014.947937

3. Schrier RW (1997) Ensuring the survival of the clinician-scientist. Acad Med J Assoc Am Med Coll 72:589-594

4. Smeesters PR (2015) You'll be a clinician-scientist, my son. J Transl Med 13:345. https://doi.org/10.1186/s12967-015-0713-8

5. DeLuca GC, Ovseiko PV, Buchan AM (2016) Personalized medical education: reappraising clinician-scientist training. Sci Transl Med 8:321fs2. https://doi.org/10.1126/scitranslmed.aad0689

6. Revet A, Hebebrand J, Klauser P (2017) The 2017 ESCAP Research Academy Workshop: bright perspectives for child and adolescent psychiatry. Eur Child Adolesc Psychiatr. https://doi. org/10.1007/s00787-017-1036-5
7. Posporelis S, Sawa A, Smith GS et al (2014) Promoting careers in academic research to psychiatry residents. Acad Psychiatr J Am Assoc Dir Psychiatr Resid Train Assoc Acad Psychiatr 38:185190. https://doi.org/10.1007/s40596-014-0037-7

8. Kennedy PGE (2015) My life as a clinician-scientist: trying to bridge the perceived gap between medicine and science. DNA Cell Biol 34:383-390. https://doi.org/10.1089/dna.2015.2860

9. Rubio DM, Schoenbaum EE, Lee LS et al (2010) Defining translational research: implications for training. Acad Med J Assoc Am Med Coll 85:470-475. https://doi.org/10.1097/ACM.0b013e31 $81 \operatorname{ccd} 618$

10. Sourander A, Chudal R (2017) The debate on child and adolescent psychiatric research is going global. Eur Child Adolesc Psychiatr 26:509-510. https://doi.org/10.1007/s00787-017-0987-x

11. Kisely S (2015) Can the next generation of clinician-scientists please step forward? Australas Psychiatr Bull R Aust N Z Coll Psychiatr 23:5-6. https://doi.org/10.1177/1039856214562123

\section{Affiliations}

\section{Alexis Revet ${ }^{1}$. Johannes Hebebrand ${ }^{2} \cdot$ Sampada Bhide $^{3}$. João Caseiro ${ }^{4}$ Eugenia Conti ${ }^{5} \cdot$ Marike Deutz $^{6}$. Andra Isac ${ }^{7}$. Athanasios Kanellopoulos ${ }^{8}$. Tuğba Kalyoncu ${ }^{9} \cdot$ Katri Maasalo $^{10}$. Silvana Markovska-Simoska ${ }^{11}$. Marija Mitkovic-Voncina ${ }^{12}$. Silvia Molteni ${ }^{13}$. Mariela Mosheva ${ }^{14}$. Susanne Mudra ${ }^{15}$. Julia Philipp ${ }^{16}$. Cecil Prins-Aardema ${ }^{17}$. Marina Romero Gonzalez ${ }^{18}$. Antonín Šebela ${ }^{19} \cdot$ Jochen Seitz $^{20} \cdot$ Lise Eilin Stene $^{21}$. Nikolina Vrljičak Davidović ${ }^{22} \cdot$ Ida Wessing $^{23} \cdot$ Paul Klauser $^{24}$}

1 Service Universitaire de Psychiatrie de l'Enfant et de l'Adolescent, CHU de Toulouse, Hôpital Purpan, Place du Dr Baylac, 31059 Toulouse Cedex 9, TSA 40031, France

2 Department of Child and Adolescent Psychiatry, Psychosomatics and Psychotherapy, LVR Klinikum Essen, University Hospital Essen, University of Duisburg-Essen, Essen, Germany

3 School of Psychological Sciences, The University of Melbourne, Melbourne, Australia

4 Departamento de Pedopsiquiatria e Saúde Mental da Infância e da Adolescência, Centro Hospitalar do Porto, Porto, Portugal

5 Department of Clinical and Experimental Medicine, University of Pisa, Pisa, Italy

6 Department of Child and Adolescent Studies, Utrecht University, Utrecht, Netherlands

7 Child and Adolescent Psychiatry and Addictions Clinic, Children's Emergency Hospital, Cluj-Napoca, Romania

8 Mental Health Care Unit "Evgenidion Therapeftirion", National Kapodistrian University of Athens, Athens, Greece

9 Tepecik Research and Training Hospital, Izmir, Turkey

10 Child Psychiatry, Children's Hospital, University of Helsinki and Helsinki University Hospital, Helsinki, Finland

11 Macedonian Academy of Sciences and Arts, Skopje, Macedonia

12 Belgrade University Faculty of Medicine, Institute of Mental Health, Belgrade, Serbia
13 Department of Brain and Behavioral Sciences, Child Neuropsychiatry Unit, University of Pavia, Pavia, Italy

14 The Child Psychiatry Division, Edmond and Lily Safra Children's Hospital, Sheba Medical Center, Tel Hashomer, Israel

15 Department of Child and Adolescent Psychiatry, Psychotherapy und Psychosomatics, University Medical Center Hamburg-Eppendorf, Hamburg, Germany

16 Department of Child and Adolescent Psychiatry, Medical University of Vienna, Vienna, Austria

17 Department of Family Psychiatry and Department of Child and Adolescent Psychiatry, Beilen, The Netherlands

18 Neuroscience Department (CIMES), Institute of Biomedical Research of Malaga (IBIMA), Malaga, Spain

19 National Institute of Mental Health, Klecany, Czech Republic

20 Department of Child and Adolescent Psychiatry, Psychotherapy and Psychosomatics, University Hospital, RWTH University Aachen, Aachen, Germany

21 Norwegian Centre for Violence and Traumatic Stress Studies (NKVTS), Oslo, Norway

22 KBC Split, Split, Croatia

23 Department of Child and Adolescent Psychiatry, Psychosomatics and Psychotherapy, University Hospital Münster, Münster, Germany

24 Department of Psychiatry, Centre Hospitalier Universitaire Vaudois, Lausanne University Hospital (CHUV), Lausanne, Switzerland 